\title{
Aviation civile et terrorisme : naissance et enjeux d'une politique européenne de sûreté des transports aériens
}

Yann Poincignon

\section{(2) OpenEdition \\ Journals}

Édition électronique

URL : http://journals.openedition.org/conflits/1632

DOI : $10.4000 /$ conflits. 1632

ISSN : 1777-5345

Éditeur :

CCLS - Centre d'études sur les conflits lilberté et sécurité, L'Harmattan

\section{Édition imprimée}

Date de publication : 1 décembre 2004

Pagination : 83-119

ISBN : 2-7475-7598-X

ISSN : 1157-996X

\section{Référence électronique}

Yann Poincignon, «Aviation civile et terrorisme : naissance et enjeux d'une politique européenne de sûreté des transports aériens », Cultures \& Conflits [En ligne], 56 | hiver 2004, mis en ligne le 07 janvier 2010, consulté le 30 mars 2021. URL : http://journals.openedition.org/conflits/1632 ; DOI : https:// doi.org/10.4000/conflits. 1632

Ce document a été généré automatiquement le 30 mars 2021.

Creative Commons License 


\title{
Aviation civile et terrorisme : naissance et enjeux d'une politique européenne de sûreté des transports aériens ${ }^{1}$
}

\author{
Yann Poincignon
}

1 Au sein des sociétés contemporaines dont une des caractéristiques essentielles est la mobilité des individus, les attentats du 11 septembre sont venus rappeler la fragilité et la vulnérabilité des réseaux de transports aériens. La sûreté des transports aériens ${ }^{2}$ représente donc un enjeu symbolique de premier ordre, compte tenu de la forte visibilité des catastrophes et attentats aériens (la diffusion des images des attentats en direct, puis en boucle sur toutes les télévisions du monde a vraisemblablement contribué pour une large part à structurer tant la riposte américaine que les réactions nationales et internationales), ainsi que du nombre souvent élevé de victimes (là encore, une sorte de paroxysme appelant une réaction immédiate est atteinte). De ce fait, on constate que la sûreté aérienne, perçue comme une composante majeure de la sécurité intérieure par les Etats membres et les institutions de l'Union européenne, occupe une place primordiale au sein de la politique de lutte contre le terrorisme de l'Union européenne.

2 Mais l'analyse de l'émergence d'une politique européenne de sûreté de l'aviation civile au lendemain des attentats dépasse la simple analyse rationnelle en termes de « réponse ». En effet, l'ampleur du choc appelait une réaction légitime des instances nationales et internationales en charge de la sécurité. Mais il importe pour bien en saisir les enjeux d'en identifier tous les acteurs et leur position dans des jeux politiques complexes à la fois nationaux, inter-gouvernementaux et communautaires qui font intervenir des acteurs publics et privés. Les Etats se voient de fait ici dessaisis d'une partie de leur gestion de la sécurité par des instances européennes (notamment la Commission qui se sert de l'argument sécuritaire pour élargir son domaine de compétence) et internationales en charge de la sûreté de l'aviation civile. De plus, les 
acteurs privés tant du domaine aéronautique (aéroports et compagnies aériennes) que du secteur de la sécurité vont jouer un rôle important en pesant sur certaines décisions. Enfin, cette question revêt un aspect classique de politique étrangère en impliquant les Etats de destination des vols: de manière plus claire encore après le 11 septembre, l'avion passe du statut de cible à celui d'arme par destination, puis par extension de cheval de Troie, qui permet d'importer de la menace à la sécurité intérieure. Ainsi, un Etat comme les Etats-Unis, qui se perçoit comme menacé au premier chef, considère-t-il légitime de chercher à se protéger au plus loin de ses frontières, en exportant ses normes sécuritaires et en les imposant à la faveur d'un rapport de force économique favorable. Pour la même raison, la politique de sûreté des transports aériens va de manière naturelle s'intégrer à celle du contrôle des mouvements des individus, en mettant l'accent sur des solutions de contrôle global, fondé sur des solutions technologiques et une mise en réseau de l'information.

3 Il semble donc ici utile de s'interroger sur la manière dont ces questions sont abordées par les institutions et les Etats. Pour cela, on caractérisera tout d'abord la gestion de la sûreté aérienne depuis la prise en compte de sa dimension internationale, afin de discerner les mécanismes et l'originalité de la politique européenne qui émerge après le 11 septembre. L'analyse des acteurs, de leur positionnement et de leurs intérêts au sein de ce débat permettra ainsi de dégager les enjeux d'une telle approche, qui contribue pour partie à façonner ce que sera la notion de sécurité européenne.

\section{La sûreté aérienne : les limites de l'approche internationale}

4 Malgré une récurrence des actes de violence à motivation politique touchant l'aviation civile depuis la fin des années 1950, la communauté internationale s'est toujours refusée à lier ces actes à la notion de terrorisme, notamment en raison de l'impossibilité d'arriver à toute forme de consensus dans la caractérisation de ce type de violence. De ce fait, la coopération internationale dans ce domaine a jusqu'ici essentiellement visé à prévenir des actes de nature criminelle et à améliorer les conditions de poursuite et de jugement de leurs auteurs. La sûreté des transports aériens a donc longtemps été uniquement régulée par des recommandations non obligatoires faites aux Etats membres par des organisations internationales. La mise en œuvre en était assurée au niveau national par la collaboration des agences de sécurité internes et externes avec les acteurs publics et privés en charge de la sécurité. Résultant d'une approche concertée au sein d'organisations intergouvernementales, la sûreté aérienne a reposé durant les cinquante dernières années sur des conventions internationales qui ne faisaient pas appel à la notion de terrorisme. Les auteurs d'actions illégales étaient poursuivis et traités comme des criminels.

Créée par une Convention signée le 7 décembre 1944, l'Organisation de l'Aviation Civile Internationale (OACI) devient en 1947 une agence spécialisée des Nations Unies. Mais son action en matière de sûreté ne devient effective qu'au début des années 1960 et revêt deux formes bien distinctes. L'organisation va en effet dans un premier temps servir de cadre, à partir de 1963, à l'élaboration de conventions pénales visant à harmoniser la coopération judiciaire dans la répression des actes illicites commis à l'encontre de l'activité aérienne. D'autre part, l'OACI va développer sa capacité d'acteur direct de la sûreté, en se dotant d'instances permanentes dédiées à cette question 
(création en 1969 du Comité de l'intervention illicite, organe auxiliaire permanent du Conseil). L'extension de la capacité normative de l'OACI au domaine de la sûreté se manifeste ainsi par la promulgation de normes impératives et de pratiques recommandées, par le biais de dispositions annexées à la Convention de Chicago. Ainsi, l'annexe 17 fixe les responsabilités des Etats en s'attachant à l'organisation des procédures nationales administratives et techniques, aux mesures préventives de sûreté et à la gestion de la riposte.

6 A la fois organisation indépendante et intégrée à l'OACI au terme de l'article 55 de la Convention de Chicago, la Conférence européenne de l'aviation civile ${ }^{3}$ (CEAC) reprend les missions de l'OACI pour sa zone géographique, en se fixant un objectif dépassant une simple perspective technique. En effet, au-delà de l'harmonisation des politiques et pratiques de ses membres, elle vise également à l'exportation de ses standards vers les autres régions du monde. Cette approche plus politique semble traduire une volonté de se positionner par rapport à l'Union européenne dans un domaine commun aux deux organisations. Cela apparaît clairement dans le discours de la Conférence, qui

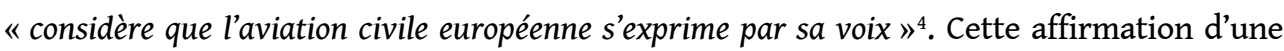
légitimité supérieure à celle de l'UE se retrouve dans la mise en valeur du consensus nécessaire à toutes les décisions de la CEAC, opposé au processus communautaire considéré comme contraignant. De même, sa portée géographique (souvent présentée comme plus grande que celle de l'Union européenne) et l'association systématique du secteur privé à ses travaux (compagnies aériennes, opérateurs aéroportuaires, constructeurs) concourent également à cette expression d'une légitimité renforcée.

7 Pour traiter de la sûreté, la CEAC a mis sur pied un groupe de travail, auquel sont subordonnées deux équipes composées d'experts nationaux ${ }^{5}$ et privés, qui traitent respectivement des questions opérationnelles et techniques. Ce groupe a pour principale mission la mise jour du Manuel de recommandations et de résolutions de la CEAC (ou Document 30), adopté en 1985. Dans ce domaine, la spécificité de la CEAC par rapport à l'OACI est double. En effet, on constate d'une part une plus grande réactivité de l'organisation européenne, qui a pu faire adopter rapidement un certain nombre de mesures techniques notamment en matière de suivi des bagages. Cela peut en partie s'expliquer par l'intégration en amont de la prise de décision d'experts privés qui poussaient à l'adoption en tant que normes de pratiques déjà en vigueur (leur caractère désormais obligatoire permettant un partage des coûts avec les Etats chargés de l'application de ces normes). En ce sens, la CEAC, en constituant un forum consultatif moins formel, veut se situer à l'avant-garde de nouvelles politiques européennes en matière d'aviation civile. De plus, la Conférence cherche à se positionner comme conseil en matière de sûreté au-delà de ses propres frontières. En effet, au nom du principe selon lequel la sûreté des installations et des aéronefs européens commence au point de départ des avions, elle encourage ses membres à faire appliquer ses standards dans les pays en développement avec lesquels ces derniers ont des relations privilégiées. Dans cette optique, des "memorandums of understanding" ont été signés avec les Commissions africaine, latino-américaine et arabe de l'aviation civile.

8 L'Union européenne, si elle a acquis un rôle de premier plan, via l'action de la Commission, dans le domaine du transport aérien et particulièrement de la libéralisation du marché, a été particulièrement absente en matière de sûreté. Plusieurs raisons peuvent à cet égard être invoquées. Tout d'abord, du fait d'une coopération croissante entre agences de police et assimilées en charge de la sécurité sur les 
installations aéroportuaires (polices, douanes, gendarmerie), la lutte contre les actes illicites à l'égard du transport aérien n'est pas apparue comme une priorité de la Commission. De plus, malgré un certain nombre d'actes touchant des compagnies, installations ou ressortissants d'Etats membres de l'Union, la remise en cause de la souveraineté étatique que traduirait une législation communautaire ne semble pas encore acceptable. La Commission européenne considérait donc cette législation comme inutile avant le 11 septembre, considérant que les Etats membres avaient réagi de manière appropriée aux vagues de terrorisme des années 1970 et 1980 dans le cadre de leur coopération au sein de l'OACI et de la CEAC. Elle prônait alors la coopération au cas par cas avec les Etats tiers, considérant que la menace était souvent liée à la nationalité des transporteurs et au lieu de départ ou d'arrivée des vols. Toutefois, une amorce de centralisation au sein de la Direction générale énergie et transports (DG TREN) des activités clefs liés à l'exploitation de l'aviation civile, excluant encore la sûreté, se dessine dès les années 1990. En effet, au-delà de la libéralisation du marché, les initiatives lancées en matière de gestion de l'espace aérien (projet « ciel unique ») et d'environnement traduisent une politique active d'élargissement des compétences du Commissaire européen et de la DG en charge de ce dossier.

Volet complémentaire de l'action directe des organisations intergouvernementales, l'élaboration de Conventions ${ }^{6}$ visant à la répression ${ }^{7}$ des actes portant atteinte à la sûreté des transports aériens sont une étape importante dans la coopération internationale contre le terrorisme. En effet, le caractère symbolique à la fois en termes de puissance économique et de souveraineté de l'avion de transport va en faire une cible privilégiée dans les années 1960 et 1970. Mais l'arsenal juridique qui sera développé en réaction à ces actions, s'il s'adaptera aux évolutions des formes de cette violence, en ignorera les motivations politiques pour s'attacher au résultat des actes. Le fait de considérer ainsi les actes de "piraterie aérienne" (cette terminologie journalistique consacrée à la fin des années $1960^{8}$ illustre bien le caractère plus criminel que politique de l'action) obéit en fait à une double motivation. La première, d'ordre démocratique, est invoquée par les Etats occidentaux pour lesquels seuls des actes peuvent être sanctionnés, et pas des opinions. Le crime est ici commis à l'égard de personnes et de biens civils, et n'est pas considéré comme «terroriste» en tant que tentative de subversion de l'ordre politique. Mais la seconde raison tient à l'efficacité même des Conventions. En effet, la qualification d'un crime comme politique entraîne automatiquement un jugement moral et ouvre donc la possibilité d'une certaine forme de reconnaissance. A l'inverse, l'inclusion d'un crime dans l'ordre du droit commun met hors-la-loi son auteur, suscitera une unanimité plus forte et permettra par là même une meilleure coopération internationale dans sa répression. Ainsi, les termes de terrorisme, de terroriste ou d'acte terroriste ne sont pas employés dans ces Conventions (seule la Convention de Montréal de 1991 emploie le terme de " terrorisme " dans ses attendus, mais sans en donner de définition, et surtout sans l'utiliser dans les articles ou pour qualifier des actes ou situations qui y sont visés). Mais l'homogénéité apparente de cette approche doit être nuancée par l'influence des acteurs privés au sein de ce processus et la limite à l'action que représente la souveraineté des Etats. 


\section{Des pratiques largement privatisées}

$10 \mathrm{Au}$ plan international, les intérêts privés sont représentés par deux associations professionnelles majeures que sont l'International Air Transport Association (IATA) et la Airport Council International (ACI) ${ }^{9}$, dont l'action se fait particulièrement sentir au sein des organisations internationales. En effet, le rôle principal des organisations intergouvernementales consiste à édicter des normes et à les faire respecter, en laissant la responsabilité d'au moins une partie du financement et de la mise en œuvre des systèmes de sécurité à la charge des compagnies aériennes et des opérateurs aéroportuaires. Il est donc vital pour ces opérateurs de faire valoir leurs intérêts au sein des instances internationales, dans les domaines touchant tant à l'exploitation qu'à la sécurité.

11 Ayant à sa création en 1945 un champ d'action limité au conseil lors de la résolution de crises et à sa participation aux enquêtes postérieures aux actes illicites, l'IATA a très rapidement élargi son mandat en se posant comme le principal conseiller technique de l'OACI lors de l'élaboration de ses résolutions. Son action fut de promouvoir systématiquement la prise en compte des aspects économiques dans la réflexion internationale sur la sûreté, les mesures prises par les compagnies lui apparaissant plus immédiatement génératrices de sécurité que les efforts des gouvernements.

12 Au-delà des aspects techniques, l'influence des compagnies aériennes en matière de sécurité se retrouve dans la volonté d'IATA de se donner un rôle plus politique. En effet, la réponse de l'OACI aux actes de terrorisme contre l'aviation civile est essentiellement une réponse nationale, à la fois par la mise en œuvre de mesures de prévention et par la répression judiciaire fondée sur l'extradition, et donc le jugement par un Etat. A l'inverse, l'IATA a tenté d'internationaliser cette réponse en proposant au Sommet de l'OACI de juin 1988 la création d'une force de réaction internationale aux détournements d'avions (combinant acteurs policiers et militaires), d'une équipe d'enquête internationale ainsi que d'une cour pénale ad hoc pour juger les auteurs d'actes commis à l'encontre de l'aviation civile. Mais l'application de la réglementation internationale passe également par une privatisation des pratiques, tant au niveau de la conception que de la mise en œuvre au sein des Etats. En effet, ces derniers délèguent la responsabilité de la sûreté à une autorité chargée de la préparation et de la mise en œuvre de plans nationaux et locaux. Si dans certains Etats de l'Union européenne, cette responsabilité est confiée à un ministère ou une agence gouvernementale, on note que pour la majorité des membres, cette compétence de conception est soit partagée entre le secteur public et l'opérateur aéroportuaire, soit confiée en totalité à ce dernier ${ }^{10}$.

Par ailleurs, le secteur public délègue majoritairement la mise en œuvre des mesures de sûreté au secteur privé. Cela se traduit principalement par le remplacement progressif des services étatiques (police et douanes) par des opérateurs de sécurité privés pour les tâches de contrôle des passagers et des bagages, ainsi que des accès aux zones protégées. Les modalités de ce transfert sont variables, les compagnies de sécurité étant soit intégrées aux sociétés d'exploitation aéroportuaire (cas du Royaume-Uni), soit indépendantes - et en général multinationales - comme le sont les trois géants européens Sécuritas, Sécuricor et ICTS. Cette délégation de certaines fonctions a pris en Europe des proportions importantes, les opérateurs privés pouvant représenter de 85 à 90 pour cent des personnels de sécurité1 ${ }^{11}$. Mais l'introduction d'une logique de marché dans ce domaine n'est pas sans soulever des problèmes d'efficacité. En effet, la sécurité 
est dans ce cas perçue, au-delà de la protection des personnes et des biens, comme un bien produit et consommé de manière à générer un sentiment de sécurité, et ainsi favoriser l'activité économique. Le caractère commercial des compagnies de sécurité est donc ici central. Cette logique de profit et de privatisation a amené la plupart de ces sociétés à diminuer leurs standards de recrutement, et à ne remplir que très partiellement leurs contrats en termes de formation et d'effectifs. De plus, une gestion sociale très précaire (salaires peu élevés, temps partiel, absence de représentation syndicale spécifique) a entraîné un turn-over extrêmement élevé (de l'ordre de 50 pour cent par an en Europe, il atteint 100 à 200 pourcent par an aux Etats-Unis). Si la qualité et la fiabilité individuelles des personnels ne sont pas remises en cause en Europe (tous font l'objet en France d'une double accréditation administrative par le préfet et judiciaire par le procureur de la République), la qualité de la prestation rendue par ces sociétés a été largement critiquée dans les années 1990.

Le secteur privé intervient également par la fourniture d'équipements, dont l'importance n'est pas à négliger. En effet, à la différence des prestations de services, le besoin de sécurité se matérialise dans ce cas dans un produit purement commercial, objectivation de la sécurité dans une réponse technologique qui est particulièrement sensible dans le domaine de la sûreté aérienne. Ainsi, face aux limitations de l'approche par le droit international, l'OACI présente dans son discours la technologie comme étant la meilleure solution pour venir à bout des actes illicites. Et les entreprises l'ont bien compris, qui se positionnent pour répondre à cette demande des opérateurs et des Etats, à l'exemple de SAGEM qui introduit la page "sûreté aéroportuaire » de son site Internet par le constat de l'insuffisance des systèmes de sécurité actuels du fait de la complexification du trafic aérien. Afin de promouvoir ses produits face à ce besoin, elle adopte une politique très active au sein de PROAVIA (association française pour la promotion des équipements et services aéroportuaires) et est membre à part entière d'ACI Europe (groupes de travail « facilitation » et « sûreté ») et d'IATA.

Afin d'asseoir leur positionnement, ces entreprises commerciales de sécurité, qu'elles fournissent un service ou des technologies, vont tenir un discours de légitimation de leur action qui leur est nécessaire dans un domaine traditionnellement réservé à l'Etat. De fait, ces entreprises réussissent à présenter leurs intérêts économiques comme étant conformes à la recherche de l'intérêt général en mettant en avant leur légalisme et leur intégrité ${ }^{12}$. Ainsi, après une phase de méfiance des pouvoirs publics à l'encontre de ce marché, un vecteur puissant de légitimation de ces acteurs en France a été l'augmentation de leur emploi dans la surveillance de lieux publics menacés par des attentats terroristes dans les années 1990. A l'inverse, les autorités publiques, avec le soutien d'organisations internationales (OACI, Conseil de l'Europe) vont mettre en avant la nécessité du recours à ce type d'opérateurs commerciaux en reprenant une partie de leur discours. La plus-value des sociétés de sécurité par rapport aux services publics en termes de flexibilité et d'adaptabilité aux variations de flux est ainsi souvent soulignée, et leur action toujours légitimée par l'argument du contrôle de l'Etat sur leurs opérations ${ }^{13}$. Ainsi, se dessine une nouvelle organisation sociale de la fonction de sécurité, dont l'interprétation nécessite de dépasser la problématique, centrée sur le rôle de l'Etat, qui consiste à se demander si la privatisation marque un retrait ou un renforcement de ce dernier. La représentation dans laquelle Etats et économie fonctionnent comme deux systèmes antagonistes doit être nuancée par la prise en compte des convergences d'intérêts évoquées ci-dessus, qui donnent lieu à des alliances 
et à certaines formes de répartition du travail, de coopération et d'échanges de ressources entre acteurs ${ }^{14}$.

\section{Une universalité inachevée}

16 Mais malgré cette constante évolution des normes et pratiques recommandées par l'OACI, et le caractère juridiquement contraignant au regard du droit international de ces instruments, on peut constater que leur application est loin d'être universelle.

Si l'argument invoqué par l'OACI du manque de coordination entre Etats et organisations internationales et du faible niveau de compétence technique de certains Etats dans ce domaine est certainement recevable, il semble nécessaire de dépasser cette vision statocentrée de l'organisation internationale. On peut ainsi se demander si les Etats ont encore les moyens politiques de faire appliquer ces normes, dans le sens où leur emprise sur les opérateurs privés auxquels ils ont délégué la sûreté va en diminuant. Cette désétatisation de la sécurité et l'introduction d'une logique de profit implique un changement radical de perspective: les intérêts poursuivis par des entreprises finançant directement des mesures de sécurité peuvent diverger de ceux d'un Etat faisant fonctionner une police agissant théoriquement pour le «bien commun $»^{15}$. En effet, les études sur le financement de la sûreté de l'aviation civile montrent clairement que les coûts sont dans la majorité des cas à la charge des gestionnaires d'aéroports et des compagnies aériennes, qui les répercutent sur les passagers. Ainsi, des entreprises soumises à une logique de marché sont donc à même de moduler, en fonction de leurs impératifs et de leurs intérêts économiques, le budget alloué à la sûreté et donc le niveau des mesures prises. Un conflit d'intérêts est donc susceptible d'apparaître entre des recommandations publiques et des intérêts privés. Le coût exorbitant de mise en œuvre, non pris en compte par les Etats et dépassant les capacités des opérateurs privés, semble donc constituer un handicap majeur à la mise en œuvre des normes internationales. L'effectivité de la Convention de Chicago est donc naturellement remise en cause par certains de ceux qui en sont les signataires. Mais au-delà des normes techniques de la Convention de 1944, c'est l'efficacité de l'ensemble de la réponse fondée sur le droit international public, y compris les conventions à portée judiciaire, qui est mise en cause.

Les conventions portant sur la répression judiciaire des actes illicites commis à l'encontre de l'aviation civile se heurtent en effet à l'obstacle de la souveraineté des Etats, qui vient constituer une limite à la fonction législative et régulatrice des organisations internationales. En effet, les Etats sont libres d'adhérer ou non aux conventions multilatérales. De fait, même si le taux d'adhésion aux Conventions de Tokyo, La Haye et Montréal est important, un certain nombre d'Etats qui n'en sont pas signataires restent en dehors de toute coopération internationale, et seront donc systématiquement recherchés par les auteurs d'actes illicites (on citera ici le cas du détournement en 1984 d'un appareil Koweïtien, dont les auteurs ne furent pas poursuivis au motif que leur Etat de destination - l'Algérie - n'était partie à aucune de ces conventions).

De plus, dans le cas où un Etat partie refuserait de livrer les auteurs d'un acte illicite, il n'existe aucune procédure ni dans les conventions multilatérales, ni dans les traités bilatéraux, autre que les sanctions classiques du droit international. De fait, rien qui permettrait de dissuader un Etat qui pour des motifs politiques refuserait de considérer 
une telle action comme délictueuse. En effet, ces instruments juridiques ont une force inférieure aux traités d'extradition (auxquels ils peuvent uniquement servir de base si les Etats le souhaitent), qui sont eux-mêmes, malgré leur caractère impératif, soumis à la volonté des Etats de les appliquer. Enfin, les obligations faites aux Etats de rechercher, arrêter et juger les suspects éventuels sont relatives. En effet, malgré l'absence de définition du terrorisme dans les conventions, la question du rapport entre crime à motivation politique et droit d'asile s'est systématiquement trouvée posée. L'échec de cet aspect de l'appareil juridique international est patent: moins de 10 pourcent des auteurs de détournements d'aéronefs commis entre mars 1968 et juillet 1974 furent emprisonnés ou tués lors d'interventions. A cet égard, certains Etats, confrontés à des difficultés dans la répression d'actes commis contre leurs compagnies ou leurs ressortissants, ont souhaité dès 1978 renforcer le caractère coercitif de la législation internationale. La déclaration lors du Sommet du G7 à Bonn en 1978 visait ainsi, malgré la position exprimée par l'OACI en 1971 contre l'introduction de sanctions dans ce domaine, à interdire l'escale aux compagnies relevant des Etats signataires dans les pays ne coopérant pas à un niveau suffisant dans l'application des accords internationaux en matière de sûreté aérienne.

L'analyse de l'évolution de la sûreté aérienne depuis 1945 révèle donc un secteur spécifique, bien démarqué de la coopération policière et de la lutte contre le terrorisme. C'est en revanche une approche radicalement différente qui est perceptible dans les mesures européennes post 11 septembre, tant dans le discours sur la menace que dans les pratiques qui reflètent le poids important des acteurs traditionnels de l'anti-terrorisme dans l'Union.

\section{Une réponse européenne qui reflète les structures institutionnelles}

21 Présentée du fait de son caractère symbolique et médiatique comme l'un des domaines majeurs d'application des mesures de lutte contre le terrorisme, la sûreté de l'aviation civile a été évoquée au sein de l'ensemble des instances européennes après le 11 septembre. Mais si le souci légitime de réduire le risque pour les usagers est présent, les jeux et les positions des différents acteurs révèlent également une double stratégie de positionnement interne des institutions et des Etats entre eux et externe de l'Union européenne par rapport aux autres organisations. L'analyse de la réaction de l'Union européenne aux attentats du 11 septembre en matière de sûreté de l'aviation civile révèle une architecture complexe, reflétant une complémentarité à la fois institutionnelle (la coprésidence Conseil - Commission instaurée pour ces débats fait intervenir des mécanismes communautaires et intergouvernementaux) et thématique (débat transports et Justice et affaires intérieures - JAI). Les intérêts et positions des acteurs impliqués seront donc intrinsèquement liés à leur place et leur importance dans ce réseau.

Le Conseil Transports est la première instance à réagir dans le domaine de la sûreté dès le 14 septembre, en prenant deux mesures principales. D'une part, le Conseil décide l'élaboration d'un document à l'attention de l'OACI, auquel pourront s'associer les membres de la CEAC qui le souhaitent, et contenant notamment une révision de l'annexe 17 en réponse aux «nouvelles menaces" ainsi qu'une proposition de programmes d'audits. De plus, il invite la Commission à créer un groupe de travail ad 
hoc multidisciplinaire, coprésidé par ces deux institutions, et chargé à la fois d'examiner les besoins en coordination et coopération dans l'introduction de nouvelles mesures de sûreté dans l'Union et de proposer les mesures législatives nécessaires à leur application effective et uniforme. La coprésidence, au-delà d'une nécessaire coordination entre domaine communautaire et intergouvernemental, reflète la volonté de la Commission et des Etats de se positionner comme acteurs incontournables d'une politique émergente. En effet, le 11 septembre voit la Commission se saisir en urgence du domaine de la sûreté pour lequel, aux dires mêmes de ses experts, elle n'était pas préparée : il importe donc pour les Etats et les représentants des agences nationales en charge de la sûreté de ne pas voir leurs prérogatives tomber intégralement dans le domaine communautaire.

Mais les mesures prises par l'Union pour renforcer la sûreté aérienne s'inscrivent sans équivoque dans le cadre plus général d'une politique de lutte contre le terrorisme, et impliquent donc les acteurs traditionnels issus de la coopération policière. Ainsi, dès le Conseil JAI extraordinaire du 20 septembre, le thème de la sûreté des transports aériens est évoqué à plusieurs titres, dont la coopération policière et des services de renseignement, les mesures aux frontières et la mise en commun de «bonnes pratiques » avec les Etats-Unis. L'ensemble de ces propositions est formalisé dans la feuille de route du 12 octobre 2001, par laquelle le Conseil JAI se voit chargé, au-delà des mesures visant à renforcer les contrôles aux frontières et dont les acteurs reconnaissent l'importance en termes de lutte contre le terrorisme dans les transports aériens, du "renforcement des normes de sécurité dans les aéroports et les avions $»^{16}$ par l'intermédiaire de l'action de la task-force des directeurs généraux de police (police chiefs operational task force, PCOTF). Tout comme la Commission s'est intéressée à des domaines touchant au titre VI dans le cadre de son action sur la sûreté aérienne, les actions en matière de sûreté des transports aériens sont à l'inverse l'occasion pour les acteurs du troisième pilier de réaffirmer de manière plus visible leur compétence dans un domaine dont ils avaient auparavant l'exclusivité. D'une manière générale, on peut distinguer deux grands types d'action, les unes ciblées sur les aéroports en tant que frontières, et les secondes visant à augmenter la sécurité des appareils en vol.

Même si les mesures concernant les frontières présentées dans la feuille de route du Conseil ne font pas directement référence à la sûreté de l'aviation civile, le lien qui est fait entre franchissement de frontière et terrorisme est particulièrement sensible dans le cas des aéroports internationaux, frontières extérieures situées à l'intérieur des Etats. En effet, ces mesures de renforcement des contrôles, complétées par le développement des réseaux d'échange d'information sur les visas et la décision de renforcer l'alimentation en signalements du Système d'Information Schengen sont perçues par les acteurs, tant policiers que membres des administrations des transports ou opérateurs privés, comme participant directement à la sûreté. La sûreté aérienne justifie donc le renforcement des pouvoirs et l'extension des missions de certaines institutions. Ainsi, la PCOTF se voit-elle explicitement confier un rôle en matière de sûreté aérienne par le Conseil JAI. Ce lien entre terrorisme et immigration est également fait au sein d'instances qui traitent de la sûreté des transports aériens. En effet, la Direction générale Transports de la Commission associe la Direction A de la DG JAI, notamment au titre de la " sous-question immigration » dont elle s'est saisie en marge du groupe ad hoc, dans le cadre de sa réflexion sur les mesures futures (notamment les techniques de vérification de l'identité des personnes et donc l'implication des acteurs 
de la sûreté aérienne dans la réflexion sur les documents d'identité et les techniques de biométrie).

Hors des institutions européennes, les Etats au sein de la CEAC ont également étroitement associé immigration, terrorisme et sûreté aérienne, relation qui s'objective dans l'existence d'un sous-groupe de travail sur l'immigration au sein du groupe de travail sur la facilitation, dont la préoccupation majeure est, selon l'organisation, «la prise en compte des impératifs de sûreté $\rrbracket^{17}$. Ce sous-groupe, présidé par le sous-directeur de l'office fédéral des étrangers de Suisse, a notamment été chargé de développer un système d'alerte sur les mouvements migratoires illégaux, ouverts aux Etats et à leurs compagnies aériennes. Ce système ECFALIS, créé à l'origine pour des raisons économiques, s'est vu relancé à l'occasion des attentats du 11 septembre, au motif que la migration illégale "compromet l'ordre et la sécurité publiques » ${ }^{18}$. De la même manière que pour la relation terrorisme - immigration, l'accent mis sur le renseignement dans la lutte contre le terrorisme va toucher les instances traitant de sûreté aérienne. Ainsi, l'Annexe 17 de l'OACI, texte jusqu'ici essentiellement technique, se voit-il renforcé de deux amendements incitant les Etats membres à échanger des informations sur les menaces pesant sur l'aviation civile. De la même manière, le groupe de travail ad hoc de la PCOTF recommande l'échange d'informations entre services de police, compagnies aériennes et services chargés de la sûreté aérienne. On constate donc ici un élargissement en termes de compétences du champ de la sécurité intérieure en Europe, les acteurs en charge de la sûreté aérienne étant ainsi incités à coopérer avec les acteurs traditionnels dans l'exécution de leur mission.

Non incluse dans le projet de règlement de la Commission, la protection des aéronefs en vol a été évoquée dans le cadre du Titre VI via une proposition autrichienne visant à l'introduction de policiers armés à bord des vols sensibles. Formés spécialement, à terme par le Collège européen de police, ces policiers seraient chargés d'empêcher les détournements, au besoin en recourant à la force, en participant aux opérations de fouille d'avion et à l'évaluation des risques que peuvent présenter les passagers. Seul Etat membre ayant recours à ce système (avec Israël, la Suisse et les Etats-Unis, qui ont fait passer le nombre de leurs "skymarshals " de 30 à 1200 après le 11 septembre), l'Autriche met ainsi en avant une expertise unique dans l'Union. Mais si quelques Etats voient cette proposition d'un œil favorable, l'évocation de ce point au sein du groupe ad hoc de la DG TREN a soulevé de profondes dissensions. En effet, de nombreux Etats, et en particulier leurs administrations policières, se sont élevés contre ce projet notamment pour des raisons d'effectifs insuffisants. De plus, la mise en œuvre de cette proposition soulèverait immanquablement la question de l'armement de gardes privés, et donc de la remise en cause du monopole de l'exercice légitime de la force armée par les agents de l'Etat. Concrètement, cette question pose enfin des problèmes de sécurité des vols, de sûreté et de responsabilité en cas de problème dans l'exécution de cette mission, responsabilité que les Etats dans leur majorité ne semblent pas prêts à accepter.

Au-delà des Etats, les compagnies aériennes, et particulièrement les syndicats de pilotes, sont particulièrement opposés à ce projet, car ces derniers souhaitent garder le monopole de la sécurité et de la sûreté du vol dont ils sont pénalement responsables ${ }^{19}$. Enfin, la question de la légitimité d'une telle action est posée, lorsque au nom de la sûreté et de la lutte contre le terrorisme, cette proposition envisage un élargissement de la mission des policiers à bord. Dans ce cadre, la lutte contre le terrorisme pourrait 
se transformer en une sorte de "droit de poursuite" déguisé permettant la continuation de l'action policière au-delà du territoire de compétence des forces de police concernées. Ces diverses oppositions ont entraîné un report de cette proposition, qui refait surface début 2004 sous forme d'une lettre confidentielle du Conseil à l'OACI. Mais si l'on constate une tendance des acteurs traditionnels de l'antiterrorisme à se saisir de la sûreté aérienne afin de légitimer leur action, on voit surtout se dessiner une autonomisation de la Commission par rapport aux Etats (et donc une entrée en concurrence directe avec la (EAC), notamment au travers de la proposition de Règlement sur la sûreté aérienne ${ }^{20}$ et de son action dans les instances internationales.

\section{Autonomisation de la Commission : le projet de règlement sur la sûreté aérienne}

La proposition de Règlement sur la sûreté de l'aviation civile marque une double rupture avec les pratiques jusqu'alors en vigueur en Europe. En effet, l'adoption d'un instrument juridique contraignant, directement applicable sans transposition dans l'ordre interne des Etats membres et pouvant faire l'objet de recours en manquement tranche avec les standards et les pratiques recommandées par les organisations internationales telles que l'OACI et la CEAC. Mais au-delà, ce texte s'inscrit résolument dans le cadre de la lutte contre le terrorisme. En effet, même si elle ne se veut que le pendant que des accords «techniques » du type Annexe 17 de l'OACI ou Document 30 de la CEAC (les décisions-cadres sur le mandat d'arrêt européen et la lutte contre le terrorisme étant elles la version européenne des Conventions de Tokyo et de Montréal), cette proposition fait explicitement référence au terrorisme, considéré comme l'une des plus graves menaces pour les valeurs fondamentales qui sont l'essence de l'Union européenne.

Le rôle de la Commission dans ce processus représente lui aussi une nouveauté. En effet, elle ne traitait pas de sûreté avant le 11 septembre, et était particulièrement mal préparée à le faire face à la CEAC. On peut ainsi noter durant le mandat de la Commission Prodi une nette tendance de la DG Transports à chercher à étendre le champ de ses compétences dans le domaine de l'aviation, avec une série de grands chantiers notamment dans le domaine du contrôle (projet "ciel unique») et de la sécurité aérienne. En revanche, l'existence de la CEAC et sa légitimité comme producteur de normes en matière de sûreté constituaient un obstacle à toute initiative de la Commission, qui aurait été ressentie comme une atteinte à la souveraineté des Etats dans un domaine non expressément visé par les Traités. Mais les événements du 11 septembre ont permis un repositionnement des acteurs. La Commission, en arguant d'une globalisation de la menace, peut ainsi se poser en point de convergence des problèmes de sûreté, qui ne peuvent plus être laissés à la seule appréciation des autorités locales ou nationales.

Ainsi, la conjonction d'une tendance structurelle au regroupement des compétences relatives à l'aviation à l'échelon communautaire et d'une opportunité conjoncturelle a permis à la Commission de se saisir d'un domaine qui relevait jusqu'ici de l'intergouvernemental et des politiques nationales. Techniquement, la proposition reprend sous forme d'annexe les principales dispositions du Document 30 de la CEAC, sur lequel un consensus existait déjà au sein des Etats membres de l'Union européenne (ce document ne concerne de fait que la sûreté au sol et non celle à bord des aéronefs). 
Cette annexe regroupe des mesures concernant la prévention des actes de terrorisme dans les zones aéroportuaires, en amont du décollage de l'avion (contrôle d'accès et des bagages, équipements, recrutement et formation des personnels). Mais la remise en cause de prérogatives étatiques, notamment en matière de financement et de contrôle va poser plus de problèmes et être à l'origine d'un retard considérable dans l'adoption du texte. En effet, la proposition ne tranche pas en faveur d'un financement étatique ou privé des mesures supplémentaires, ce qui a pour effet de reconduire les pratiques en vigueur (financement par les compagnies aériennes et les exploitants aéroportuaires). De même, la question des délais de mise en œuvre, intimement liée à celle des coûts pour les mesures les plus lourdes, fait l'objet d'une approche peu nuancée de la Commission qui propose que le Règlement entre en vigueur dans sa totalité dans les vingt jours suivant sa publication.

31 La souveraineté des Etats en matière de sûreté aérienne est également remise en cause par l'instauration d'un mécanisme de contrôle et d'audit obligatoire des plans nationaux de sécurité et des aéroports, mis en œuvre par la Commission. En effet, seuls les Etats eux-mêmes, par le biais de leurs administrations, avaient le pouvoir de déclencher des audits de la CEAC sur une base volontaire. De fait, il apparait que la Commission est dans ce domaine perçue par les Etats comme une «menace » à la fois à leur souveraineté et à leur réputation internationale en matière de sécurité, dans la mesure où les résultats des audits, s'ils n'ont pas vocation à être publiés, seront en revanche diffusés aux autorités compétentes des Etats membres. Au contraire du financement de la mise en œuvre des mesures de sûreté, celui des audits fait l'objet d'une annexe détaillée de la proposition de règlement. Celle-ci illustre de manière claire le processus de captation de la sûreté aérienne par la Commission et la manière dont elle entend se doter d'une capacité dans ce domaine (création d'une structure permanente d'inspection, dotée d'un budget propre).

32 Mais le principal point d'achoppement en matière de remise en cause de la souveraineté étatique réside dans l'article 6 de la proposition, qui octroie la possibilité aux Etats membres de décider de mesures plus strictes que celles préconisées, mais donne pouvoir à la Commission d'en décider le retrait si elle les estime discriminatoires, non justifiées ou excessivement restrictives. Ce point a fait l'objet d'une opposition conjointe du Parlement et du Conseil. En effet, s'il apparaît légitime que la Commission se penche sur les distorsions commerciales induites par ces mesures, la possibilité qu'elle s'octroyait de décider du caractère justifié ou excessivement restrictif des mesures a été interprétée par les acteurs étatiques comme une remise en cause du Titre VI. Certains représentants y voyaient même un début de communautarisation masquée de la coopération policière, avec l'institution d'un droit de veto de la Commission sur les pratiques policières des Etats.

33 Enfin, le choix même de la voie réglementaire par rapport à une directive qui aurait pu voir des transpositions divergentes dans les droits nationaux est révélateur de la volonté de la Commission de contrôler le processus d'européanisation de la sûreté aérienne par le biais de la procédure de comitologie. En effet, le groupe ad hoc de la Commission deviendra avec l'entrée en vigueur du Règlement un comité réglementaire, chargé de prendre les mesures additionnelles nécessaires à l'efficacité de la législation communautaire ainsi que celles concernant la mise en place des audits. S'il est toujours composé de représentants des Etats membres éventuellement renforcés d'experts (sans droit de vote), ce comité, assisté d'un sous-groupe «mesures futures» convoqué de 
manière ad hoc, passera de fait sous la présidence unique de la Commission qui en fera l'instrument de promulgation des mesures d'application du Règlement. Les stratégies du Conseil et de la Commission sont à l'égard du projet de Règlement fort différentes : en effet, le Conseil a cherché dès le départ à en diminuer la portée en le rendant plus détaillé, afin d'éviter toute dérive communautaire par la suite. Pour sa part, la Commission met en avant la nécessité d'une autonomie très large ainsi que de la mise en place de moyens de contrôle sur les politiques menées par les Etats. Mais si la Commission remet en cause les prérogatives nationales des Etats, elle les concurrence également dans leur action au sein des organisations internationales de l'aviation civile.

\section{La Commission européenne et les organisations internationales de l'aviation civile}

34 La Commission européenne va donc utiliser sa capacité normative par la voie réglementaire, plus rapide que l'élaboration d'une convention ou l'obtention d'un consensus au sein de la CEAC, pour se positionner face à cette dernière. La production juridique devient ainsi un facteur de légitimation d'une politique.

35 Au-delà d'une simple captation d'un champ de compétence, on peut voir ici une tentative d'instrumentalisation de la CEAC par la Commission européenne au nom d'une légitimité plus forte. En effet, si la proposition originale de la Commission ne visait qu'à transposer le Document 30 dans l'ordre interne de l'Union, les mesures additionnelles vont plus loin en reprenant l'ensemble des études menées par la Conférence. Aux dires mêmes des fonctionnaires de la Commission, la CEAC est un organe dont l'utilité est incontestable en tant que rassemblement des expertises et la proposition de solutions techniques, mais qui ne doit jouer qu'un rôle de promotion de la sûreté au-delà de l'UE, le rôle politique et normatif étant dévolu à la Commission. Face à cette position communautaire, la CEAC invoque quant à elle le manque de compétence technique en matière de sûreté de la Commission (qui n'a pas participé substantiellement à l'élaboration du Document 30 malgré sa position d'observateur à la CEAC, et qui n'employait pas d'experts à titre permanent). De plus, elle met l'accent sur l'unilatéralisme dans la prise de décision communautaire, qui n'associe pas les acteurs privés à son élaboration alors que ces derniers ont souvent à supporter le financement des mesures de sûreté.

La CEAC cherche également à se présenter comme une institution politique, en mettant l'accent sur le déficit de légitimité de l'Union européenne. Le discours de la Conférence souligne en effet le caractère obligatoire et non consensuel des normes adoptées par la Commission, en y opposant la manière consensuelle et non directive dont elle traite de problèmes abordés de manières diverses par les Etats (qui confient la sûreté à des acteurs divers allant des opérateurs privés aux ministères de la Défense, en passant par l'Intérieur ou la Justice). Cette approche différenciée est particulièrement sensible dans la définition de la sûreté. Pour la Commission, il s'agit d'un problème essentiellement technique qui peut être résolu par des mécanismes communautaires et par l'implication minimale du Titre VI TUE ${ }^{21}$. A l'inverse, la CEAC met en avant le caractère politique de la notion de sûreté, avec notamment l'implication très en amont des spécialistes $d u$ renseignement ${ }^{22}$. De ce fait, elle reconnaît que les règles de confidentialité sur un sujet aussi sensible doivent différer de celles qui sont applicables 
en matière de sécurité aérienne, notamment dans la publicité donnée aux rapports d'inspection.

La CEAC va donc se positionner face à la Commission en réaffirmant son rôle central en matière de sûreté par la création de trois task-forces spécialisées, chargées d'apporter une contribution au travail communautaire. Les missions de ces groupes, parallèles à ceux de la Commission, soulèvent deux remarques. D'une part, on voit la CEAC se saisir d'un domaine au sujet duquel la Commission affiche quelque réticence car il semble relever plus de la coopération policière que de la simple coopération technique, à savoir la présence de gardes armés à bord des appareils. De plus, l'accent mis sur les audits permet aux Etats de regagner un pouvoir que les mécanismes communautaires leur font perdre: si la Commission continue à s'inspirer des acquis de la CEAC, l'élaboration de programmes et de procédures d'audits par consensus au sein de cette organisation intergouvernementale présente un grand intérêt pour les Etats qui sont plus libres dans ce type de négociation (le terme même d'audit continue à faire l'objet de débats, le Règlement visant à le remplacer par le terme de monitoring ou inspections). Les approches des deux organisations sur cette question des audits sont divergentes dans leur esprit. En effet, la CEAC se fonde sur le volontariat et la confidentialité, alors que la Commission souhaite l'instauration d'audits obligatoires, dont les résultats seront connus des administrations des Etats membres. Le risque existe donc de voir se développer deux circuits d'audits parallèles, qui seraient euxmêmes partiellement redondants avec les mécanismes nationaux et ceux que l'OACI prévoit d'instaurer. En effet, l'organisation mondiale se positionne également dans ce marché de plus en plus compétitif de la sûreté aérienne, non plus en tant qu'instaurateur de normes (dont on a vu les limites dans un cadre aussi large), mais en légitimant son action par l'instauration de contrôles : ainsi a été décidée à Montréal en 2002 la mise en place d'audits réguliers, obligatoires, systématiques et harmonisés des plans nationaux et des aéroports sans précision sur les liens prévus entre ces contrôles et ceux déjà existants.

Enfin, la représentation de l'Union européenne au sein des organisations internationales (CEAC et OACI) est un enjeu d'importance pour la Commission. Mais les pratiques varient et les intérêts s'affrontent dans ce domaine, où l'on retrouve un débat classique sur l'expression de la politique étrangère d'un acteur international: son élaboration et sa défense au sein d'instances internationales doit-elle être confiée à des diplomates (Conseil, Présidence en exercice) ou à des spécialistes du domaine considéré (Commission) ? La dérive vers le second cas de figure constatée au niveau des politiques étrangères des Etats se retrouve ici au niveau européen. Actuellement, la représentation de l'Union à l'OACI est assurée par la Présidence en exercice, qui a présenté à Montréal en février 2002 une position commune de l'UE et de la CEAC adoptée par le COREPER. Mais pour la Commission, qui a rédigé cette proposition de texte commun, le Conseil - et encore moins la Présidence en exercice, qui tourne tous les six mois - n'a pas la compétence pour défendre cette position au sein de l'OACI. De plus, la majorité des points abordés ressortant - selon les fonctionnaires de la Commission - du domaine communautaire, il y a là un dessaisissement et qui ne pourrait se résoudre que par la représentation de l'UE à l'OACI par la Commission au détriment de la Présidence. 


\section{L'engagement des acteurs économiques : le dilemme coût/ efficacité}

39 L'argument économique du maintien de l'activité économique et de la restauration de la confiance est invoqué par l'ensemble des acteurs pour justifier la politique de lutte contre le terrorisme dans les transports aériens. Ainsi, la Commission dans son évaluation générale des conséquences du 11 septembre et des mesures prises par l'Union ne sépare absolument pas les problèmes de sûreté aérienne des conséquences en termes de compétitivité. Cette concurrence des intérêts de sécurité et des intérêts économiques liés au financement des mesures se reflète d'ailleurs dans la disparité des situations en Europe concernant le financement des mesures de sécurité après le 11 septembre. Dans leur grande majorité, les opérateurs aéroportuaires et les compagnies aériennes ont donc dû supporter le coût de ces mesures exceptionnelles, sans pouvoir par ailleurs toujours les répercuter sur les utilisateurs.

40 La Commission se refuse donc à évoquer les problèmes de financement, laissant aux Etats la responsabilité de légiférer dans ce domaine, incitant ainsi les opérateurs privés à modifier leur approche de la sûreté. En effet, les groupements internationaux tels qu'ACI ou IATA ne voyaient pas le renforcement des mesures d'une manière favorable avant le 11 septembre, en raison de leur impact négatif sur la facilitation (le ralentissement des flux) ainsi que du coût de leur mise en œuvre. Ne pouvant plus tenir ce discours revendiquant une privatisation de la sécurité après le 11 septembre, ces opérateurs privés rappellent les gouvernements à leur « devoir impérieux ». Pour cela, ils utilisent un argument moral, en appelant les Etats à la restauration de leurs prérogatives régaliennes et les mettant devant leurs responsabilités. Cette position s'appuie sur une interprétation particulière de la menace. En effet, ces acteurs considèrent que le 11 septembre a démontré que le transport aérien n'a été qu'instrumentalisé par les terroristes pour s'attaquer aux Etats; en aucun cas il n'était une cible en soi, et donc ne peut être tenu de financer l'ensemble des mesures de sécurité qui visent en fait à protéger l'ensemble des citoyens et non plus les seuls utilisateurs. D'autre part, une pression est exercée sur les gouvernements par l'invocation du contexte de crise financière dû à la réduction du trafic des passagers et à l'augmentation des primes d'assurance. Dans ce cadre, une augmentation importante des charges de sécurité représente un risque pour la survie même du secteur de l'aviation civile.

41 L'influence des groupements professionnels internationaux sur les institutions européennes en matière de sûreté diffère des mécanismes habituels pour l'aviation civile. En effet, le point d'entrée classique de ces acteurs, qui possèdent des représentations à Bruxelles, est la Commission, qui les consulte et les associe à de nombreuses réunions techniques. Mais dans la mesure où cette dernière n'a pas intégré les questions de financement dans sa proposition, et n'a pas fait initialement appel à ces opérateurs privés pour débattre de ces problèmes au sein du Groupe ad hoc ${ }^{23}$, ces organisations se sont tournées vers le Parlement européen, traditionnellement attaché au financement équitable des mesures d'application de la législation communautaire. On note ainsi que les amendements proposés par le Parlement en première et en seconde lecture de la proposition de Règlement sur la sûreté aérienne reflètent les intérêts des compagnies aériennes et des opérateurs aéroportuaires, en demandant leur étroite association à toute procédure, notamment en matière de financement. Il 
s'agit en effet d'une part de diminuer les coûts en supprimant ou en allégeant certaines mesures de sûreté, ou en les étalant dans le temps. Mais la principale demande tant du Parlement que des professionnels est, au nom des arguments précédemment évoqués et contre l'avis de la Commission, de faire supporter par les Etats une partie des coûts des mesures permanentes et exceptionnelles. Il est toutefois à noter que la Commission et le Conseil se sont opposés à la quasi-totalité de ces propositions. En effet, celles concernant la diminution des coûts sont perçues comme tendant à réduire le niveau de sûreté, tandis que celles visant à rendre les Etats financièrement responsables des mesures de sûreté sont considérées comme n'entrant pas dans le champ de compétence du Règlement. De plus, on comprend que les Etats, au travers de leur vote au Conseil, s'opposent à une réglementation qui leur est défavorable. De ce fait, les problèmes de financement représentent les principaux points de discorde au cours des réunions de conciliation, et très certainement responsables de l'impossibilité de faire adopter avant l'été 2002 un règlement qui devait aboutir en décembre 2001.

42 Au-delà des aspects financiers, une demande importante du renforcement du rôle de l'Etat se manifeste également au niveau de la mise en œuvre des mesures de sûreté, et particulièrement en ce qui concerne le recours aux opérateurs privés de sécurité. Ce débat, particulièrement animé aux Etats-Unis, a vu un consensus entre Républicains et Démocrates pour dénoncer l'échec d'un système privé ultra-libéral, et aboutit à la création d'un corps fédéral de sûreté des transports de 28000 fonctionnaires. Principalement fondée sur la nécessité d'uniformiser les pratiques et de mettre un terme aux logiques de profit suivies par certaines compagnies dont les standards étaient jugés par trop déficients, cette proposition s'est rapidement vu objecter que le système précédent ne pouvait se voir imputer la responsabilité des attentats du 11 septembre (les individus n'étaient pas répertoriés comme dangereux, et il était à l'époque légal d'introduire à bord les instruments utilisés comme armes lors de ces attentats). Au sein de l'Union européenne, le débat ne s'est pas posé de manière aussi tranchée, mais plutôt en termes de convergence d'intérêts, à organiser au mieux en matière de responsabilité et de contrôle. En effet, la proposition de Règlement de la Commission ne vise pas uniformiser les pratiques en matière de responsabilité de sécurité, invoquant simplement la notion, reprise par le Conseil, «d'autorité compétente». Cette autorité désignée par les gouvernements, qui peuvent ainsi perpétuer les pratiques antérieures, peut donc être soit publique soit privée.

Le contrôle exercé sur les opérateurs privés dans l'exercice de leur mission semble quant à lui poser plus de questions, en particulier par la difficulté à réglementer dans ce domaine. En effet, ce point est l'objet d'une concurrence assez vive entre les institutions du troisième pilier (notamment le groupe de coopération policière) qui souhaitent être saisies du dossier au nom du contrôle de la police sur les opérateurs privés, et la Commission qui veut voir ce problème traité sous couvert de la liberté d'entreprise, ce qui lui permet en outre de conforter son rôle dans le domaine de la sécurité. Le Parlement européen (Commission libertés publiques) a sur ce point demandé à la Commission d'élaborer une proposition de directive d'harmonisation de l'emploi des services de sécurité privée, qui est à l'étude conjointement par les DG Marché Intérieur et JAI. La nécessité de coopération entre Etats et secteur privé est également avancée par le Parlement européen, notamment en matière d'accréditation et de formation des personnels. Face à la Commission et au Conseil qui souhaitaient voir les personnels habilités par leur employeur, la Commission parlementaire des Transports a clairement affirmé son souhait de voir les administrations nationales 
prendre la responsabilité de ces accréditations. De même, un certain nombre d'amendements ont été déposés, appuyés notamment par les opérateurs aéroportuaires, visant à restreindre le nombre de personnels concernés par une formation minimale obligatoire.

Mais d'une manière générale, la Commission reconnaît l'importance du secteur privé de la sécurité dans la fiche d'évaluation d'impact associée à sa proposition de Règlement, en prévoyant que les entreprises spécialisées en matière de sûreté pourront connaître une certaine expansion. De fait, on constate en Europe une tendance inverse à celle observée aux Etats-Unis, avec une augmentation massive du nombre des employés privés dans les aéroports, justifiée principalement par une plus grande flexibilité tant sur le court terme (temps partiel en fonction des horaires de pointe) que sur le long terme (possibilité d'embauche en période de crise et de réduction plus facile des effectifs en période calme ${ }^{24}$. Cette augmentation ne concerne d'ailleurs pas uniquement les agents d'exécution. En effet, on voit certains exploitants mettre sur pied des corps de contrôle et d'encadrement des opérateurs de sécurité (250 personnes dans le cas d'Aéroports de Paris), chargés à la fois de contrôler la bonne application des marchés et de compléter l'encadrement des compagnies de sécurité privées par les agents du service public.

La politique de sûreté des transports aériens qui se met en place dans l'Union européenne se démarque donc à plusieurs titres des pratiques antérieures au sein d'enceintes intergouvernementales comme l'OACI ou la CEAC. En ayant recours à des instruments juridiques contraignants, et surtout en intégrant cette politique à une politique plus globale de sécurité intérieure, l'UE met ainsi l'accent sur la sécurisation des frontières et le contrôle des mouvements de population qui sont au cœur du débat sur la sécurité intérieure. Mais au-delà, se pose la question de l'influence de l'acteur majeur, tant sur le plan économique que de la sécurité, que sont les Etats-Unis. En effet, l'administration américaine a usé de pressions politiques et économiques pour faire prévaloir sa vision de la sûreté aérienne et par extension de la sécurité aux frontières.

\section{Les enjeux politiques : quelle vision de la sécurité ?}

L'utilisation des techniques biométriques dans le filtrage des mouvements transfrontaliers dans les aéroports est recommandée par un nombre croissant d'acteurs de la sûreté aérienne, essentiellement au nom d'une plus grande fiabilité. Cette question se pose dans les aéroports à un double niveau. En effet, cette pratique est recommandée pour se substituer aux badges d'accès des personnels aux zones sécurisées, sans poser de réel problème. Mais sa généralisation sert d'argument pour justifier de l'instauration de tels contrôles sur les passagers eux-mêmes. Dans ce cas, il s'agit d'instaurer des passeports ou des cartes d'identité biométriques pour les passagers acceptant de se soumettre à un contrôle de sécurité préalable et unique, ou de faire apposer, puis contrôler une empreinte digitale sur les billets d'avion. En dehors des conséquences que de tels procédés peuvent avoir sur la liberté de circulation des individus, ils posent de manière fondamentale des questions sur la protection de la vie privée en autorisant le stockage et la mise à disposition d'informations personnelles dans des conditions encore mal définies ${ }^{25}$. L'utilisation qui peut être faite de ces informations doit également être analysée. En effet, des mesures de contrôle exceptionnelles se justifient au nom d'une menace majeure sur la sûreté aérienne et de 
la prévention d'actes terroristes. Mais leur détournement toujours possible à des fins répressives pour des délits mineurs, tant du fait d'une traçabilité accrue des mouvements que de possibilités renforcées d'identification des individus, soulève la question de la proportionnalité de la réponse à la menace.

Les pressions exercées en faveur du renforcement de l'emploi de ces techniques sont de deux ordres. En effet, elles émanent des entreprises détentrices du savoir-faire dans ce domaine, dont le discours est souvent repris par les acteurs politiques. Mais dans le cas de l'Union européenne, les Etats-Unis tendent également à promouvoir une politique de contrôle via le recours aux technologies de surveillance, comme en témoignent les demandes directes adressées par le gouvernement américain au Président de la Commission en octobre 2001. Ces demandes, si elles se heurtent à la réticence de la Commission et de certains Etats au nom principalement de la sauvegarde des libertés individuelles, n'en sont pas moins reprises par certaines autres instances politiques, tel le Conseil de l'Europe, dont l'Assemblée tient un discours très volontariste en matière de recours aux technologies de sécurité.

Mais dans le domaine du filtrage des individus aux frontières, un contrôle systématique ne peut être envisagé, même s'il repose sur les technologies les plus sophistiquées. Ainsi, ce recours aux technologies avancées comme la biométrie demande lui-même à être optimisé par des logiques proactives de désignation des groupes à risque au travers notamment du profilage. Dans le domaine de la sûreté des transports aériens, cette technique d'identification des individus à risque a notamment été mise au point par la compagnie israélienne $\mathrm{El} \mathrm{Al} \mathrm{en} \mathrm{coopération} \mathrm{avec} \mathrm{les} \mathrm{services} \mathrm{de} \mathrm{sécurité} \mathrm{de} \mathrm{ce} \mathrm{pays.} \mathrm{Elle}$ consiste, au travers de questionnaires rapides, à sélectionner des individus potentiellement dangereux pour les aiguiller vers des équipements de détection plus performants. Mais cette technique repose sur une double condition non dénuée de risques. En effet, elle suppose tout d'abord une coopération poussée entre l'Etat et les compagnies aériennes, opérateurs aéroportuaires et de sécurité privée dans l'exécution de mesures de sécurité qui dépassent le simple contrôle de bagages : elles impliquent un contrôle de l'individu au travers de questions personnelles. Se pose ainsi la question de la légitimité de telles pratiques, notamment de la part d'acteurs privés. Ce procédé suppose en outre que la menace soit clairement définie, ce qui est loin d'être toujours le cas dans le cadre des mesures souvent réactives mises en place après des attentats. Il va donc contribuer à légitimer, par l'implication même d'acteurs privés et des passagers se soumettant aux contrôles, la définition institutionnelle de la menace. Ainsi, au-delà d'une privatisation des actions de sécurité, apparaît donc également le risque d'une privatisation du discours sur la menace, les agents en charge des contrôles se trouvant investis du pouvoir de catégoriser et de désigner "l'ennemi» potentiel. Mais si la surveillance peut s'exercer de manière directe sur les individus lors de leur passage de la frontière, elle va également devenir plus globale en cherchant à utiliser aux fins de la lutte contre le terrorisme l'ensemble des moyens existants de collecte, d'échange et de traitement de l'information. Ce lien entre accès à l'information et pouvoir de contrôle trouve ici tout son sens, au travers de l'importance donnée au renseignement dans le cadre de la lutte contre le terrorisme, en particulier par l'utilisation des moyens technologiques les plus avancés, tels que les réseaux de fichiers informatisés.

49 L'utilisation extensive de ces fichiers informatiques comme moyen de contrôle au nom de la lutte contre le terrorisme revêt plusieurs formes. Elle peut se traduire par l'ouverture de certains d'entre eux, comme le SIS, à des agences telles que les services 
de renseignement ou Europol. Dans le cas de l'agence policière, la première phase envisagée, qui prévoit l'accès aux informations, lui permettra de passer outre les réticences de certains Etats à fournir des renseignements, et pourrait donc voir ces derniers perdre confiance dans ce système et refuser de continuer à l'alimenter en invoquant une utilisation trop large des informations qu'ils fournissent. De plus, la question d'un accès total ou limité est posée. En effet, un accès exhaustif verrait de fait Europol avoir accès à des informations qui ne sont pas de sa compétence, comme par exemple au titre de l'article 96 de la convention de Schengen, relatif aux étrangers non admissibles. Dans ce cas, l'utilisation par un organe de police judiciaire de données de cas relevant de la police administrative des étrangers, matière en laquelle elle n'a pas compétence, pose question tant au plan juridique que sur celui des libertés individuelles. Mais au-delà des avantages opérationnels qu'Europol tirerait de cet accès en termes de traçabilité des individus, l'agence avance également une raison stratégique d'évaluation, qui lui permettrait de fait de participer de manière encore plus active à la définition de la menace, notamment au travers de la détermination de tendances temporelles, géographiques, ethniques et démographiques.

La seconde phase de cet accès prévoit la possibilité pour Europol d'introduire et de mettre à jour des données dans le système. Or, dans la mesure où les Etats ne bénéficient de ce droit de modification que pour les données dont ils sont propriétaires, l'agence de police européenne serait ainsi la seule administration détentrice d'un pouvoir central sur ce fichier. Ainsi, l'extension des fonctionnalités d'un fichier dont le contrôle démocratique est sujet à débat (seule une autorité de contrôle créée par la Convention est saisie de cet aspect, à l'exclusion du Parlement européen et des parlements nationaux) pourrait se voir entérinée sans modification préalable de la Convention qui l'institue. En parallèle à cette extension de l'accès aux fichiers existants, on constate également la création de nouveaux réseaux de données, à l'image du système ECFALIS mis en place par la CEAC. Mais l'utilisation de ces fichiers peut rapidement, au nom de la prévention d'une menace vitale, dépasser le cadre initialement prévu. Ainsi, selon le responsable de ce projet qui relaie ainsi l'opinion de certains Etats et opérateurs privés, " la question se pose de savoir si une limitation d'ECFALIS aux Etats membres de la CEAC et aux méthodes de mouvements migratoires est toujours adéquate $»^{26}$. Ainsi, s'impliquant plus avant dans la coopération policière et l'échange de renseignements, la CEAC va au-delà d'une action sur le contrôle de la migration au nom de la sûreté. Elle renforce ainsi le caractère politique de son action, face à une Commission qui veut la cantonner dans un rôle plus technique.

51 Cette logique proactive, illustration du mythe du contrôle global des mouvements comme réponse à une menace importée, a également des ressorts externes à l'Union européenne. Elle est en effet largement appuyée par l'administration américaine, qui au nom de l'efficacité antiterroriste prône une homogénéisation des pratiques, revenant de facto à exporter les normes américaines dans des Etats tiers. En effet, les demandes américaines à l'Union tendent à imposer une vision globale et technologique de la surveillance, en incitant en particulier les Etats européens à revoir leur position. Elles invitent en particulier l'UE à reconsidérer sa politique de protection des données dans le contexte des impératifs de la lutte contre le terrorisme (incluant la surveillance d'Internet), ainsi qu'à réviser les textes exigeant la destruction des «données opérateurs » au-delà d'un certain délai. 


\section{Europe et Etats-Unis : deux visions divergentes de la sécurité ?} européennes ne saurait s'interpréter uniquement comme la résultante de jeux internes à l'Union. Ainsi, la réaction immédiate de fermeture de l'espace aérien par les EtatsUnis après le 11 septembre, ainsi que les positions dures prises par ce pays au sein des institutions internationales vont nettement façonner la réponse européenne, au premier chef pour des raisons d'ordre économique. En effet, il apparaît d'une part nécessaire de susciter la confiance américaine dans le système européen afin de préserver le trafic transatlantique. En effet, l'argument économique est le premier à revenir dans les motivations ${ }^{27}$ des différents acteurs. D'autre part, il importe également, compte tenu de la tendance à l'action unilatérale des Etats-Unis dans ce domaine, d'aligner les pratiques européennes en matière de sûreté afin de ne pas créer de distorsions commerciales préjudiciables aux exploitants ${ }^{28}$. renforcement des mesures au-delà des recommandations de l'OACI, s'exerce d'abord de manière directe. En effet, la liste des demandes américaines transmise au président de la Commission le 16 octobre 2001, et notamment ses chapitres sur la protection des données, les contrôles aux frontières et la sûreté aérienne, dépasse les mesures envisagées par l'Union. Elle se trouve même sur certains points (comme par exemple la protection des données) en contradiction avec des points considérés comme fondamentaux par les institutions européennes. Mais cette pression est également indirecte au sein de l'OACI et d'autres organisations généralistes. A cet égard, le G8 représente un forum de choix pour l'expression des vues américaines, dans la mesure où le poids économique de cet Etat fait que ses positions sont difficilement discutables. De fait, les mesures adoptées par les membres du G8 au Sommet de Kananaskis en juin 2002 reflètent de manière particulièrement fidèle les mesures prises au niveau national par Washington. C'est donc bien à une exportation de la vision de la sécurité des EtatsUnis vers l'Europe que l'on assiste dans ce domaine, vision qui reflète la politique développée par Washington dans sa lutte contre le terrorisme. A l'image du concept de "Homeland security ", le discours institutionnel européen met l'accent sur l'importation de la menace et la notion de sécurité «intérieure ». Ainsi, la lutte contre le terrorisme est pensée en termes de frontière et de maitrise des flux. Cette perspective se dessine notamment au travers du recours grandissant, pour exercer ce contrôle, aux technologies de pointe, mises en avant par les acteurs qui en ont la maîtrise. De ce fait une nouvelle géographie se dessine, opposant de manière plus marquée un intérieur à sécuriser et un extérieur dont il faut se protéger.

Par ailleurs, la coopération européenne avec des Etats-Unis est grandement facilitée, voire amplifiée, par la qualité des relations "techniques» entre praticiens de la sécurité. En effet, la volonté du Conseil JAI de mettre en commun les bonnes pratiques dans le domaine de la lutte antiterroriste, notamment dans le domaine des technologies d'identification et de filtrage, se voit portée par une culture de la technologie commune de part et d'autre. De plus, cette culture était déjà très présente chez les professionnels de la sûreté aérienne, dont le discours et les méthodes semblent ici avoir été repris par les acteurs policiers. Ainsi, on voit l'Union européenne - sous pression américaine et contre la volonté initiale des Etats, des institutions et des populations - accélérer ce 
processus de mise en place de moyens de contrôles des mouvements de plus en plus sophistiqués. Ainsi, le recours aux technologies de filtrage et d'identification des individus et l'interconnexion des systèmes d'information, s'ils étaient à l'ordre du jour au sein de l'UE, se heurtaient jusqu'ici à des oppositions d'ordre administratif et éthique de la part de certains Etats membres et du Parlement. Le développement du recours aux empreintes biométriques pour l'établissement de pièces d'identité, ainsi que la communication au bureau américain des douanes et de l'immigration de données fournies par les passagers aux compagnies lors de l'achat de leur billet (Passenger Name Record ou PNR), sont à cet égard révélateurs.

Le recours aux techniques biométriques se développe dans l'Union après le 11 septembre. En effet, malgré les avis défavorables donnés par différentes autorités nationales, la Commission a proposé courant 2003 deux projets de Règlements visant pour l'un à imposer des documents de visa et de séjour de ce type aux ressortissants des pays tiers pour 2005 , et pour l'autre des documents d'identité à empreintes biométriques aux ressortissants de l'Union pour une date non précisée. Faisant suite à l'adoption post 11 septembre de visas et de permis de séjour à sécurité renforcée (comprenant notamment des photographies) en 2002, cette nouvelle étape a été conduite à l'initiative du Benelux et de l'Allemagne au sein du Conseil JAI, notamment sous la présidence grecque. Pour sa part, la Commission s'est fait le relais des Etats qu'elle a soutenus, en légitimant la position de l'Union par les demandes américaines imposant des passeports à données biométriques avant l'automne 2004. Le lien entre lutte contre le terrorisme et contrôle de l'immigration est ici encore très présent. En effet, si l'un des arguments avancés est d'empêcher la falsification des documents, l'harmonisation entre les informations biométriques et les différents systèmes d'information est également à l'ordre du jour. Il s'agit donc ici, via le nouveau Système d'information des Visas, regroupant des systèmes nationaux et un système central européen, d'échanger des informations sur l'ensemble des étrangers soumis à autorisation entrant et sortant de l'Union.

Cette mise en réseau de l'information sur la circulation des individus pose donc la question de la protection des données, tant au sein des Etats qu'au niveau de l'Union. En effet, les systèmes nationaux varient d'un Etat à l'autre, et si tous les Etats membres sont dotés d'une autorité de contrôle, les effectifs et les budgets qui leurs sont consacrés ne sont pas homogènes. Par ailleurs, les pouvoirs conférés à ces organismes sont également variables, certains étant dotés de véritables pouvoirs d'investigation alors que d'autres sont limités au contrôle a posteriori des actes législatifs. Au plan européen, la protection des données individuelles est régie par une directive datant de 1995. Mais si ce texte est fréquemment invoqué, notamment par la Commission dans ses propositions relatives au stockage et au transfert de données personnelles, il est loin d'être universellement efficace. Le fait qu'il ne s'agisse que d'une directive - qui nécessite par conséquent une transposition en droit national pour être appliquée - fait reposer en fin de compte ce contrôle sur les Etats, avec leurs perceptions différentes. Ainsi, un rapport de la Commission paru en 2003 sur l'application de ce texte fait ressortir des manquements dans sa mise en œuvre, et critique le manque d'indépendance de certaines de ces instances de contrôle.

La pression des Etats-Unis est également significative dans le domaine de la transmission d'informations relatives aux passagers par les compagnies aériennes (PNR). Pour des raisons essentiellement économiques, l'Union va tendre à un 
alignement avec les Etats-Unis dans ce domaine. Ces fichiers PNR sont créés par les opérateurs aériens lors des réservations et permettent à tous les intervenants du secteur aérien d'avoir accès aux informations concernant le voyage (vols aller et retour, historique des vols précédents, correspondances, services requis, repas spécifiques, problèmes de santé...). Mais si l'utilisation de ces données par les opérateurs afin de veiller au bon déroulement du voyage est légitime, leur transmission à des services de police ou d'immigration à des fins de profilage et de catégorisation semble plus problématique. Or, l'administration américaine en a très rapidement autorisé l'accès après le 11 septembre au travers de deux textes: l'Aviation and Transportation Security Act du 19 novembre 2001 et le Enhanced Border Security and Visa Entry Reform Act du 5 mai 2002. Ces deux textes, rappelés dans une demande aux compagnies aériennes européennes en mars 2003, imposent la transmission de données PNR (en tout plus d'une trentaine) aux autorités américaines, sous peines de sanctions très lourdes, pouvant aller jusqu'à une amende de 6000 dollars par passager ou même l'interdiction pure et simple d'atterrissage.

Au niveau européen, les réactions sont diverses. Au travers de la Direction générale Marché intérieur, compétente en matière de protection des données, la Commission européenne a rapidement souligné les risques potentiels d'un tel transfert de données relatives à la vie privée, et sa contradiction avec la législation européenne existante. Mais si elle s'est attachée à négocier et mettre en place un encadrement juridique de ce transfert, elle a sur le fond reconnu le bien-fondé des demandes américaines et en a accepté le principe. Le Parlement, traditionnellement attaché à la défense des libertés publiques et à l'autonomie de décision de l'Union et de ses Etats membres s'oppose à de nombreuses reprises à ce projet (notamment dans deux Résolutions extrêmement critiques des 13 mars et 9 octobre 2003). Par ailleurs, un groupe de travail créé par la directive de 1995 sur la protection des données («Groupe de l'Article 29») s'est exprimé régulièrement en critiquant ouvertement ce projet.

Ce groupe estime que l'utilisation qui pourrait être faite de ces données risquerait de dépasser le cadre de la lutte contre le terrorisme. En effet, dans une société démocratique, une situation d'exception peut justifier des limitations temporaires et strictement encadrées de certaines libertés fondamentales pour la sauvegarde de l'intérêt public. En revanche, leur légitimité est plus contestable dans le cas d'une autre utilisation comme la contribution au contrôle de l'immigration ou des missions de police, missions qui ne sont pas clairement exclues par les textes américains. De plus, le champ des données communicables apparait trop vaste compte tenu des besoins et de ce qui est considéré comme "nécessaire, proportionné et non excessif» par la directive de 1995. Sur ce point, la Commission a arrêté avec les autorités américaines une liste de 34 points, dont 20 ne sont pas acceptables aux yeux du groupe de l'Article 29. Enfin, la méthode de transfert des données pose la question de la souveraineté des Etats membres. En effet, l'agence américaine réclame un transfert de type «pull», qui lui garantirait un accès direct aux fichiers des compagnies, que la Commission envisage d'accorder, constatant que le système de type "push» par lequel ces opérateurs transfèreraient eux-mêmes les données n'existe pas. Mais ces transferts de données posent également la question du contrôle de l'utilisation qui en sera faite une fois transmises à l'administration américaine. Il semble en effet qu'elles pourront, sous couvert des textes régissant la sécurité intérieure et qui ont permis la mise en réseau de plusieurs agences fédérales, être transférées à différents services de police et de renseignement. En particulier, l'agence de sécurité des transports (TSA) rattachée au 
nouveau département de la sécurité intérieure, a mis en place un système automatisé de profilage des passagers, le système CAPPS II (Computer Assisted Passengers PreScreening System). Ce système, croisant des informations de provenance diverse et pour partie secrètes (demandes de visa, passeports, y compris des données biométriques ?), attribue à chaque passager un code couleur vert, jaune ou rouge en fonction du risque potentiel qu'il représente. Le but affiché par la TSA est d'élargir au maximum la provenance des données injectées dans ce système afin d'en accroître la fiabilité, le transfert des données PNR à la TSA étant considéré comme essentiel.

Ainsi, parallèlement à une mise en réseau de l'information sur les mouvements des individus au sein des Etats de l'Union une interconnexion des réseaux européens et américains se met en place. Et de fait, ce lien se fera d'autant plus facilement que les données relatives à ces mouvements seront informatisées par des acteurs privés (PNR) ou publics (documents de voyage numériques, données biométriques...). Ainsi, les organismes en charge de la sécurité des frontières, et notamment de celles où ces contrôles sont techniquement réalisables comme les aéroports, disposeront-il de moyens avancés de catégorisation et de profilage. L'utilisation qui sera faite de ces systèmes, et notamment de systèmes entièrement automatisés de catégorisation des individus comme CAPS II, se révèle un enjeu capital, étant donné la relative faiblesse des instances de contrôle nationales et européennes. Ce renforcement de la politique de sécurité intérieure européenne par captation d'un nouveau domaine d'action communautaire en matière de sûreté aérienne soulève donc un certain nombre de questions quant à la vision de la sécurité européenne qu'il participe à définir. En effet, les attentats du 11 septembre appelaient une réaction des institutions et des Etats européens, tant pour répondre à une menace accrue pesant sur leurs citoyens que pour rassurer une opinion publique et un secteur économique particulièrement marqués par ces évènements. Cependant, l'accélération de la production administrative et juridique dans ce domaine semble avoir éclipsé la volonté politique des gouvernants. La vision de la sécurité qui semble émerger de ce processus paraît résulter davantage des coopérations préexistantes entre les différents acteurs que d'un projet global. Or, une politique, fut-elle de sécurité, ne saurait se substituer au projet politique qui doit l'inspirer. En créant l'illusion d'une politique de sécurité efficace à cent pour cent, l'Union européenne encourage des pratiques sécuritaires qui remettent paradoxalement en cause les valeurs qui la fondent, comme la liberté de circulation des individus et d'autres libertés liées à la protection de la vie privée. A l'inverse, la préservation de ces valeurs nécessite un projet politique cohérent, duquel dérive une approche globale de la sécurité. Rapportée à la prévention et la répression des actes terroristes, cette vision se doit de prendre en compte tant les causes que les modes d'action du terrorisme, tout en insistant en permanence sur le caractère exceptionnel, temporaire, proportionnel et limité des mesures sécuritaires. Par la définition d'un tel projet, l'Europe doit pouvoir éviter l'instrumentalisation de la lutte contre le terrorisme comme facteur de légitimation, une guerre qui ne serait, selon l'expression de Jean Baudrillard ${ }^{29}$ que la "continuation de l'absence de politique par d'autres moyens ». 


\section{NOTES}

1. Cet article reprend et prolonge un travail de DEA mené sous la direction de Didier Bigo à l'Institut d'études politiques de Paris en 2002, auquel on pourra se reporter pour des références bibliographiques plus précises. Les propos exprimés ici n'engagent que leur seul auteur.

2. Par sûreté aérienne (aviation security), on entendra ici la prévention et la protection contre des actes de malveillance et des actions illicites contre l'aviation ; cette notion est complémentaire de celle de sécurité aérienne (aviation safety), qui entend la protection contre les défaillances techniques et humaines (Simon J.P., Manuel de sûreté aéroportuaire, Aéroports de Paris, 3ème édition, mars 2000).

3. Créée en 1955 sur une initiative conjointe du Conseil de l'Europe et de l'OACI, elle regroupe aujourd'hui 41 Etats dont les 25 membres de l'Union européenne.

4. Site http://www.ecac-ceac.org/index2.html, page de présentation de la CEAC.

5. Venant d'Etats membres de la CEAC ou invités pour leur représentativité (Etats-Unis) ou leurs compétences particulières (Israël).

6. La Convention relative aux infractions et à certains autres actes survenant à bord des aéronefs signée à Tokyo le 14 septembre 1963 s'applique d'une manière très large à des actes qui peuvent compromettre la sécurité de l'avion, des personnes, des biens embarqués, le bon ordre et la discipline à bord. Elle dispose que tant l'Etat du lieu où a été commis l'acte que l'Etat d'immatriculation d'un aéronef ont compétence pour connaître des infractions commises à bord et peut donc servir de base à l'extradition. La Convention pour la répression de la capture illicite d'aéronefs (Hijacking Convention) signée à La Haye le 16 décembre 1970 la complète. Elle définit l'acte de capture illicite d'un aéronef, que les Etats contractants s'engagent à réprimer de peines sévères (article 2). Elle contient des dispositions détaillées sur la compétence des Etats de connaitre les infractions, sur les poursuites et le maintien en détention des responsables et sur leur jugement ou leur extradition. La Convention pour la répression d'actes illicites dirigés contre la sécurité de l'aviation civile signée à Montréal le 23 septembre 1971 vient élargir la précédente quant aux infractions concernées. La définition d'acte illicite est étendue aux infractions commises envers les passagers et l'équipage ainsi qu'à la destruction de l'avion, et s'applique non seulement à l'aéronef en vol mais également au sol avant et après vol (et permet ainsi l'intervention au sol). De même que pour la Convention de La Haye, infraction et tentative d'infraction sont mises sur un pied d'égalité. Complémentaire de cette Convention, le Protocole pour la répression des actes illicites de violence dans les aéroports servant à l'aviation civile internationale, signé à Montréal le 24 février 1988 élargit la notion de sûreté aux installations aéroportuaires. N'entrant pas dans la catégorie des instruments de coopération judiciaire, mais néanmoins généralement citée parmi les grandes Conventions relatives à la sûreté de l'aviation civile, la Convention sur le marquage des explosifs plastiques et en feuilles aux fins de détection, signée à Montréal le 1er mars 1991, vise à faciliter la détection d'explosifs par un marquage chimique et à interdire la circulation d'explosifs non marqués.

7. On notera que le terme français de "répression " employé dans l'intitulé et le texte des conventions étudiées ici est la traduction du terme anglais « suppression ».

8. FNSP, Centre de documentation contemporaine, «L'organisation de l'aviation civile internationale », dossier de presse 9.553 A, tome 1 (1944 -1989).

9. Fondé en 1991, Airport Council International regroupait, en 1997, 475 aéroports internationaux et autorités aéroportuaires, soit environ 1200 plateformes dans 152 Etats. Son but, commun avec celui de l'IATA, est de promouvoir un transport aérien « safe, secure and efficient ».

10. Données tirées du document Financing civil aviation security costs in Europe édité le 13 mars 2002 par ACI Europe (disponible en ligne sur http://www.aci-europe.org/). Trois Etats membres de 
l'UE confient la responsabilité de la sûreté à une agence gouvernementale (DGAC en France, ministère de la Justice aux Pays-Bas, autorité conjointe ministère des Transports - ministère de l'Intérieur en Italie); quatre Etats ont instauré une compétence partagée public - privé (Danemark, Allemagne, Portugal et Royaume-Uni); six Etats confient cette responsabilité à l'opérateur aéroportuaire majeur du pays (Belgique, Finlande, Grèce, Irlande, Espagne et Suède). Les données sur l'Autriche et le Luxembourg ne sont pas disponibles.

11. Editorial, «Politicizing Airports», Wall Street Journal, 29 octobre 2001. A Amsterdam, 2000 employés privés pour 200 à 250 policiers; 450 pour 60 à Berlin; 3000 pour 20 et 1500 pour 11 à Heathrow et Gatwick; 1000 pour 200 à Paris.

12. Ocqueteau F., "La sécurité privée en France, état des lieux et questions pour l'avenir », Les Cahiers de la sécurité intérieure, n 33, 3ème trim. 1998, pp. 105-127.

13. Il est cependant à noter que cette supervision étatique est loin d'être la règle générale en Europe, où les services de sécurité sont très souvent supervisés par les gestionnaires d'aéroports.

14. Nogala D., "Le marché de la sécurité privée: analyse d'une évolution internationale ", Les Cahiers de la sécurité intérieure, $\mathrm{n}^{\circ}$ 24, 2ème trim. 1996, pp. 121-141.

15. Ocqueteau F., op. cit. p. 126.

16. Note Coordination de la mise en ouvre du plan d'action pour la lutte contre le terrorisme, 12759/01 du 12/10/01, points 41 à 45 pour les frontières et 47 pour la sûreté aérienne.

17. Périodique trimestriel d'information CEAC Info, $\mathrm{n}^{\circ} 20$, décembre 2001, p. 9.

18. Ibid. p. 10 .

19. On peut noter à ce titre l'existence de propositions antérieures - hors d'Europe - d'armer les pilotes et membres d'équipages eux-mêmes, forme particulière de privatisation de l'action policière et de la sécurité mentionnée dans un rapport du Conseil de l'Europe, Transport aérien et terrorisme : comment renforcer la sûreté ?, rapport 9296 de l'Assemblée parlementaire du Conseil de l'Europe, 18/12/01, exposé des motifs, point 19.

20. Commission des Communautés Européennes, Proposition de règlement du Parlement européen et du Conseil relatif à l'instauration de règles communes dans le domaine de la sûreté de l'aviation civile, COM (2001) 575 - 2001/0234 (COD), 10/10/2001.

21. "Le problème n'est en fait pas différent de celui qui se pose pour la sécurité aérienne et les mêmes solutions peuvent être utilisées dès lors qu'on veut garantir un niveau uniforme de protection, le plus élevé possible, à tous nos citoyens. Il suffit pour ce faire d'adopter des normes communes et de mettre en place les moyens de contrôle de leur application; la discipline communautaire et les mécanismes du Traité feront le reste ». Commission des Communautés Européennes, Proposition de règlement du Parlement européen et du Conseil relatif à l'instauration de règles communes dans le domaine de la sûreté de l'aviation civile, $\operatorname{COM}(2001) 575$ - 2001/0234 (COD), 10/10/2001, exposé des motifs, points 11 et 12.

22. Les représentants des services de police et des agences de renseignement sont conviés aux réunions des groupes de travail de la CEAC pour y participer à l'évaluation de la menace. De plus, l'exemple y est souvent cité du directeur de la sûreté du ministère britannique des Transports, qui est systématiquement un ancien fonctionnaire du MI6.

23. Soulevant ainsi leur indignation (voir notamment EWERS G., « Security developments: much haste but how much action? », Airport Business (périodique d'information d'ACI), December 2001/ January 2002, pp. 17-19). Cette position a conduit la Commission à créer un «parallel stakeholder group », comprenant les représentants des intérêts privés des compagnies et opérateurs aéroportuaires.

24. Ainsi Aéroports de Paris voit-il le nombre d'agents privés de sécurité passer de 1500 à 4000 après le 11 septembre 2001. Au Danemark, une formule originale conciliant flexibilité du privé et visibilité du public consiste à faire travailler des agents privés revêtus d'un uniforme du service public. 
25. Un projet d'Air France de mettre en place de tels dispositifs a reçu un accueil défavorable de la Commission nationale informatique et libertés, qui si elle ne s'oppose pas à l'introduction de procédés biométriques pour les personnels travaillant sur les aéroports, s'est montrée réticente à leur généralisation au contrôle des passagers.

26. Eugster R., «ECFALIS », in CEAC Info, op. cit., p. 11.

27. Jusque dans l'exposé des motifs du Conseil après l'adoption de la Position Commune relative à la proposition de Règlement sur la sûreté aérienne. Le Conseil considère en effet qu'elle vise à " renforcer la confiance dans les transports aériens en Europe » avant même d'assurer « la protection des citoyens dans la Communauté européenne, en empêchant les actes d'intervention illicite». Document 15029/4/01 REV4 ADD1 du 29/01/02, pp. 2-3.

28. Le groupe ad hoc se voit d'ailleurs confier la mission de vérifier la cohérence de la réglementation communautaire avec la législation américaine par le Conseil transports du 14 septembre.

29. . Cité dans l'article de Wieviorka M., "Réflexions sur le 11 septembre et ses suites", Confluences Méditerranée, $\mathrm{n}^{\circ}$ 40, Hiver 2001-2002, p. 39.

\section{RÉSUMÉS}

Cet article analyse la politique européenne de sûreté de l'aviation civile qui a été développée dans l'Union européenne après les attentats du 11 septembre 2001. Afin d'en appréhender les caractères originaux, il se penche dans un premier temps sur l'approche intergouvernementale qui a prévalu jusque là, notamment au travers de conventions négociées au sein d'organisations internationales. Puis il analyse la politique qui se développe dans l'Union dans le cadre du plan d'action de lutte contre le terrorisme à compter d'octobre 2001, qui reflète quant à elle les jeux institutionnels internes à l'UE, ainsi que les rapports de force entre les différents acteurs publics et privés de la sûreté. Enfin, il tente de formuler les enjeux de cette politique en termes de vision de la sécurité qu'elle véhicule, vision caractérisée par un recours important aux technologies de contrôle ainsi que par un alignement contraint sur les pratiques américaines.

This article analyses the European civil aviation security policy developed in the European Union since the September $11^{\text {th }}$ events. In order to better understand the original characteristics of such policies, the author examines the intergovernmental approach that had been since then prevailing, and in particular through negotiated conventions within international organizations. The author later analyses the developing policy with regards to the anti terrorist action plan starting October 2001 and reflecting EU internal institutional games as well as existing power games between the various security public and private actors. He eventually tries to formulate this policy's stakes in terms of the vision of security it carries, and which is characterized by the major use of control technologies and the alignment on US practices.

\section{INDEX}

Mots-clés : Aviation civile, sécurité, Union européenne, lutte anti-terroriste 


\section{AUTEUR}

\section{YANN POINCIGNON}

Yann POINCIGNONest officier de l'armée de terre, breveté de l'enseignement militaire supérieur.

Il a mené, dans le cadre du DEA de relations internationales de l'IEP de Paris, un travail de recherche sur la lutte contre le terrorisme dans l'Union européenne et la sûreté des transports aériens.Il collabore au programme ELISE. 